University of Nebraska - Lincoln

DigitalCommons@University of Nebraska - Lincoln

Ralph Skomski Publications

Research Papers in Physics and Astronomy

April 1993

New Magnets from Interstitial Intermetallics

J.M.D. Coey

Trinity College, Dublin, Ireland

Ralph Skomski

University of Nebraska-Lincoln, rskomski2@unl.edu

Follow this and additional works at: https://digitalcommons.unl.edu/physicsskomski

Part of the Physics Commons

Coey, J.M.D. and Skomski, Ralph, "New Magnets from Interstitial Intermetallics" (1993). Ralph Skomski Publications. 14.

https://digitalcommons.unl.edu/physicsskomski/14

This Article is brought to you for free and open access by the Research Papers in Physics and Astronomy at DigitalCommons@University of Nebraska - Lincoln. It has been accepted for inclusion in Ralph Skomski Publications by an authorized administrator of DigitalCommons@University of Nebraska - Lincoln. 


\title{
New Magnets from Interstitial Intermetallics
}

\author{
J. M. D. Coey and R. Skomski \\ Department of Pure and Applied Physics, Trinity College, Dublin 2, Ireland
}

Received March 29, 1993; accepted April 27, 1993

\begin{abstract}
The outlook for improving the energy product of permanent magnets beyond the theoretical limit of $516 \mathrm{~kJ} / \mathrm{m}^{3}$ for $\mathrm{Nd}_{2} \mathrm{Fe}_{14} \mathrm{~B}$ is discussed. Most promising are nanostructured two-phase magnets consisting of an aligned hard-magnetic skeleton phase possessing exceptionally high uniaxial anisotropy and a soft-magnetic phase which assures a high magnetization. Brown's paradox is discussed for inhomogeneous magnets, and micromagnetic calculations are used to predict energy products as high as $1090 \mathrm{~kJ} / \mathrm{m}^{3}$ in suitable nanostructured $\mathrm{Sm}_{2} \mathrm{Fe}_{17} \mathrm{~N}_{3} / \mathrm{Fe}_{65} \mathrm{Co}_{35}$ composites, such as multilayers or disordered two-phase magnets with common $\mathrm{c}$ axis.
\end{abstract}

\section{Introduction}

The performance of permanent magnet materials is epitomized by their energy product $(B H)_{\max }$, a figure of merit which corresponds to twice the maximum magnetostatic energy available from a magnet of optimum shape. Energy product has doubled every twelve years since the beginning of the century. It increases with coercivity $H_{c}$ and remanence $M_{\mathrm{r}}$ but can never exceed the value $\mu_{0} M_{\mathrm{r}}^{2} / 4$ corresponding to an ideal rectangular hysteresis loop. An upper limit for the remanence is the spontaneous magnetization $M_{0}$. Hence the quest for improved energy product has been a search for compounds with a large magnetization combined with the strong uniaxial anisotropy needed to develop hysteresis in a material with suitable microstructure. In former years, the problem was to achieve the necessary anisotropy, but more recently the focus has shifted to the problem of enhancing the magnetization.

The best solutions so far have been intermetallic compounds of iron or cobalt with one of the magnetic light rare earths. The $3 d$ element supplies the necessary magnetization and ferromagnetic order, whereas the rare earth to which it is coupled by exchange is chosen to furnish uniaxial anisotropy via the crystal-field interaction and spin-orbit coupling. Light rare earths are preferred because their net moments couple parallel to those of the late $3 d$ elements. Iron is better than cobalt, because it has one less electron in the $3 d$ band, and hence a larger magnetization. The pure elements have magnetizations $\mu_{0} M_{0}=2.15 \mathrm{~T}$ for $\mathrm{Fe}$ and $1.76 \mathrm{~T}$ for Co.

Unfortunately, the number of suitable compounds is very limited. First to be developed were the $1: 5$ and $2: 17$ Sm-Co alloys, since there exist no binary iron-based alloys with the necessary properties. Then in 1983 the ternary compound $\mathrm{Nd}_{2} \mathrm{Fe}_{14} \mathrm{~B}$ was discovered $[1,2]$, which has $\mu_{0} M_{0}=1.61 \mathrm{~T}$. Most high-performance magnets are now made of this material. A more recent advance was the discovery that rare earth intermetallics can be interstitially modified with nitrogen, to produce new materials with magnetic properties dramatically different from those of the parent compound [3]. The best example is $\mathrm{Sm}_{2} \mathrm{Fe}_{17} \mathrm{~N}_{3}$ which has attractive permanent magnet properties. Interstitial modification offers the prospect of tuning the Curie temperature and anisotropy, but it has rather little effect on the magnetization. The intrinsic properties of these hard magnetic materials are summarized in Table 1.

A regrettable feature of all these materials is that their magnetization is much lower than that of iron, or even cobalt. This is because the rare earth atoms occupy a volume three times as large as that of the $3 d$ element, with no commensurate contribution to the magnetic moment; $\mathrm{Pr}$ and Nd contribute $\sim 3 \mu_{\mathrm{B}}$ /atom, whereas the contribution of $\mathrm{Sm}$ is practically zero. On the other hand, the intrinsic anisotropy, represented by the anisotropy field $B_{\mathrm{a}}$, may be far greater than that required to provide the coercivity. For practical purposes there is usually no call for coercivity much greater than $M_{0} / 2$, yet historically the outcome of years of development of any new permanent magnet has been a rectangular loop with coercivity of about $20 \%$ of the anisotropy field [4]. (The anisotropy field features in the theoretical limits on the coercivity, discussed in section 4.3). The interstitially-modified compounds in particular have surplus anisotropy.

The record energy product is held by $\mathrm{Nd}_{2} \mathrm{Fe}_{14} \mathrm{~B}$ where it has been possible to achieve energy products as high as $405 \mathrm{~kJ} / \mathrm{m}^{3}$ in laboratory-scale magnets [5], quite near the theoretical limit $\mu_{0} M_{0}^{2} / 4=516 \mathrm{~kJ} / \mathrm{m}^{3}$. New approaches will have to be found if the energy product is ever to double again.

Here we focus on nanostructured two-phase magnets. The idea is to break out of the straitjacket of natural crystal structures by artificially structuring new materials on the scale of a few crystallographic unit cells. The nanocomposites will consist of a soft phase with a large magnetization, exchange-coupled to a hard phase with surplus anisotropy. The concept is similar to that of the $4 f-3 d$ intermetallics

Table 1. Intrinsic magnetic properties of permanent magnet compounds

\begin{tabular}{lclc}
\hline & $T_{\mathrm{c}}(\mathrm{K})$ & $\mu_{0} M_{\mathrm{s}}(\mathrm{T})$ & $B_{\mathrm{a}}(\mathrm{T})$ \\
\hline $\mathrm{SmCo}_{5}$ & 1000 & 1.14 & 25 \\
$\mathrm{Sm}_{2} \mathrm{Co}_{17}$ & 1193 & 1.25 & 7 \\
$\mathrm{Nd}_{2} \mathrm{Fe}_{14} \mathrm{~B}$ & 593 & 1.6 & 9 \\
$\mathrm{Sm}_{2} \mathrm{Fe}_{17} \mathrm{~N}_{3}$ & 749 & 1.54 & 21 \\
\hline
\end{tabular}


themselves, but on a different scale, where the atoms are replaced by blocks 10 to 100 atoms thick. Some encouraging experimental results have appeared on the $\mathrm{Nd}_{2} \mathrm{Fe}_{14} \mathrm{~B} / \mathrm{Fe}_{3} \mathrm{~B}$ [6] and $\mathrm{Sm}_{2} \mathrm{Fe}_{17} \mathrm{~N}_{3} / \mathrm{Fe}$ [7] systems, produced by melt spinning or mechanical alloying, respectively. Exchange coupling across an interface between hard and soft regions has been discussed by several other authors [8, 9]. Here we will derive analytic results for nucleation field and energy product of aligned two-phase magnets, and suggest how the composites with a high energy-product might best be structured. In this context, we emphasise the benefits that may be avilable from using interstitiallymodified materials, and discuss some aspects of the interstitial modification process.

\section{Intermetallic Compounds}

\subsection{Magnetic moment and magnetization}

Moment formation in magnetic materials reflects the existence of unpaired inner electrons in transition metals arising from Coulomb interaction of electrons in different orbitals. Completely filled inner shells show no net moment, whereas outer electrons tend to reduce their kinetic energy by forming bonds or bands with equal $\uparrow$ (spin-up) and $\downarrow$ (spindown) occupancy. The $3 d$ and $4 f$ elements are the ones which can offer unpaired electron density in the metallic state.

The $3 d$ electrons in a metal are delocalized, forming $\uparrow$ and $\downarrow$ bands, and the orbital component of the magnetic moment is largely quenched by the crystal potential. Observed atomic moments of $2.22 \mu_{\mathrm{B}}$ for iron and $1.72 \mu_{\mathrm{B}}$ for cobalt essentially reflect the density of unpaired electron spin. The traditional explanation of these moments, corroborated by modern band calculations [10], is that cobalt is a strong ferromagnet with a fully-polarized $3 d \uparrow$ subband, and a partly-filled $3 d \downarrow$ subband. Iron, if it behaved similarly, would have a moment of $2.72 \mu_{\mathrm{B}}$ and its magnetization would be $\mu_{0} M_{0}=2.8 \mathrm{~T}$. The smaller moment observed in practice reflects incomplete spin polarization, with electrons of either spin present at the Fermi level. Iron is therefore designated as a weak ferromagnet.

In fact, the best bulk room-temperature magnetization that has been achieved to date is $2.45 \mathrm{~T}$, in an $\mathrm{Fe}_{65} \mathrm{Co}_{35}$ alloy. The effect of alloying with cobalt is to shift some states from the lower part to the upper part of the $3 d \downarrow$ band for iron, and vice versa for cobalt. The iron moment thereby increases, while the cobalt moment is essentially unaltered [11].

Possible ways to improve the iron moment are (i) band narrowing or increased exchange splitting to move all the $3 d \uparrow$ states below the Fermi level, and (ii) charge transfer out of the $3 d$ band, provided the spin-down density of states exceeds the spin-up density. These effects are seen at their most extreme in compounds of iron with an electronegative element such as oxygen. The $3 d$ band in ferric oxides is narrowed and completely exchange-split so the ionic moment due to the fully-occupied $3 d \uparrow$ states is $5 \mu_{\mathrm{B}}$. The rest of the valence electrons are transferred to oxygen, where they form large $\mathrm{O}^{2-}$ anions, whose packing determines the density and structure of the crystal. Hypothetical ferromagnetic $\mathrm{Fe}_{2} \mathrm{O}_{3}$ would have a magnetization of only $2.5 \mathrm{~T}$ despite the
$5 \mu_{\mathrm{B}}$ iron moment, because most of the volume is occupied by oxygen. The tendency of half-filled shells to couple antiferromagnetically is an additional complication, and in fact the best that can be achieved in ferrites such as $\mathrm{MFe}_{12} \mathrm{O}_{19}$ $(\mathrm{M}=\mathrm{Ba}, \mathrm{Sr})$ is a ferrimagnetic structure where the two unequal antiparallel sublattices together with the large volume fraction of non-magnetic atoms, produce a low saturation magnetization $\left(\mu_{0} M_{0}=0.47 \mathrm{~T}\right.$ for $\left.\mathrm{SrFe}_{12} \mathrm{O}_{19}\right)$. The tendency for nearly half-full $3 d$ shells to couple antiparallel has also precluded the development of manganese-based ferromagnets with large moments.

The principal magnetic interaction in an iron-rich alloy is due to direct overlap of the $3 d$ shells of neighbouring iron atoms. That interaction is usually ferromagnetic, but it depends sensitively on the interatomic spacing and there is evidence for iron that it changes sign when the interatomic spacing exceeds about $2.5 \AA$, which is a typical Fe-Fe distance. This means that dense-packed iron-rich intermetallics, show a certain tendency towards antiferromagnetism, and their Curie temperatures are often quite low (e.g. $325 \mathrm{~K}$ for $\mathrm{Y}_{2} \mathrm{Fe}_{17}$ ).

The atomic moments vary considerably from one crystallographic site to another in iron-rich intermetallics, although this is not so for their cobalt-rich counterparts. Table II illustrates the point by comparing the iron moment and hyperfine field at the site with the largest moment in three compounds, with the values averaged over all sites.

The effects of interstitial modification on the iron sublattices are most clearly seen for the case of $\mathrm{Fe}_{4} \mathrm{~N}$. Here the nitrogen occupies the small octahedral interstitial site at the centre of the face-centred cubic crystal structure. It dilates the lattice, which makes the $\gamma$-phase ferromagnetic with $T_{\mathrm{c}}=767 \mathrm{~K}$. The nitrogen $2 p$ orbitals hybridize with the $3 d$ orbitals of their iron neighbours on the face centre sites, but there is no net charge transfer to nitrogen - if there were, the nitrogen would become too big to fit onto the cramped interstitial site. The face-centre iron sees its moment reduced by hybridization, and consequent charge transfer from the cube-corner iron which ends up with an impressive moment, but one achieved mostly at the expense of the majority, facecentre iron atoms. A zero sum game.

In this context, it is interesting to note that giant iron moments have been reported for thin films of the compound $\mathrm{Fe}_{16} \mathrm{~N}_{2}$, which has a similar structure to $\mathrm{Fe}_{4} \mathrm{~N}$ except that only half the interstitial sites are occupied. Magnetization as high as $2.9 \mathrm{~T}$ is claimed, corresponding to an iron moment of $3.2 \mu_{\mathrm{B}}$ [12]. There is no supporting evidence of exceptional magnetic moments in bulk samples containing a large

Table II. Iron moment and hyperfine field at the high moment site compared with average values in interstitial compounds

\begin{tabular}{llll}
\hline & Iron moment $\left(\mu_{\mathrm{B}}\right)$ & $\begin{array}{l}{ }^{57} \mathrm{He} \text { hyperfine } \\
\text { field (T) }\end{array}$ \\
\hline $\mathrm{Fe}_{4} \mathrm{~N}$ & 1 $a$ site & 2.98 & 36.6 \\
& average & 2.20 & 26.8 \\
$\mathrm{Nd}_{2} \mathrm{Fe}_{14} \mathrm{~B}$ & 8j $j_{2}$ site & 2.85 & 34.3 \\
& average & 2.25 & 29.5 \\
$\mathrm{Y}_{2} \mathrm{Fe}_{17}$ & $4 f$ site & 2.26 & 34.6 \\
$\mathrm{Y}_{2} \mathrm{Fe}_{17} \mathrm{~N}_{3}$ & average & 1.98 & 30.3 \\
& $4 f$ site & 2.65 & 40.1 \\
& average & 2.29 & 34.8 \\
\hline
\end{tabular}


proportion of this metastable phase [13], and at present $\mathrm{Fe}_{16} \mathrm{~N}_{2}$ must be regarded as one of the mysteries of metallic magnetism.

\section{Anisotropy and coercivity}

In the past it was necessary to resort to cumbersome bar and horseshoe shapes to avoid spontaneous demagnetization of iron magnets into a multidomain state by the magnet's own magnetostatic field. Later, shape anisotropy associated with a special metallurgical microstructure (Alnico) or the relatively weak magnetocrystalline anisotropy of $\mathrm{Fe}^{3+}$ in hard ferrites $\left(\mathrm{BaFe}_{12} \mathrm{O}_{19}\right)$ was used to create the first truly permanent magnets. Modern highperformance magnets such as $\mathrm{SmCo}_{5}, \mathrm{Nd}_{2} \mathrm{Fe}_{14} \mathrm{~B}$ or $\mathrm{Sm}_{2} \mathrm{Fe}_{17} \mathrm{~N}_{3}$ achieve much stronger anisotropy by exchange coupling $3 d$ atoms to rare-earth atoms in sites with uniaxial symmetry. There may be an additional contribution arising from the magnetocrystalline anisotropy of the $3 d$ element itself.

This means that the anisotropy of $4 f$ intermetallics is essentially derived from the electrostatic interaction of the localized $4 f$ charge cloud with the crystal field generated by the non- $4 f$ charges. The $4 f$ shell usually has a trivalent configuration. The $4 f$ shells of empty, half-filled, and filled $4 f$ shells are spherical so $\mathrm{La}, \mathrm{Gd}$, and $\mathrm{Lu}$ ions have no quadrupole moment and do not contribute to the magnetic anisotropy. The first three rare-earths of each half-shell $-\mathrm{Ce}, \mathrm{Pr}$, $\mathrm{Nd}$, and $\mathrm{Tb}, \mathrm{Dy}, \mathrm{Ho}$, respectively - exhibit oblate $4 f$ charge distributions, whereas the $4 f$ orbitals of $\mathrm{Pm}, \mathrm{Sm}, \mathrm{Eu}$, and $\mathrm{Er}$, $\mathrm{Tm}, \mathrm{Tb}$, respectively, are prolate. Subjected to a given electric field gradient, the rare-earth anisotropy contribution changes sign if a prolate ion is replaced by an oblate one, and vice versa. This principle can be used to predict the anisotropy of new intermetallics.

The electric field gradient experienced by the $4 f$ electrons in a metal is created at very short range because the conduction electrons effectively screen all distant charges. Just the immediate neighbours have to be considered, and their effect is largely to deform the outer electron shells of the rare earth to which they bond [14]. The interaction of the $4 f$ quadrupole moment with the electric field at the rare earth is the leading, second order term in the expression for the crystal field interaction at the rare earth.

In interstitially-modified intermetallics, the interstitial atoms such as nitrogen or carbon preferentially occupy large $R_{2} T_{4}$ octahedral interstices between two rare earth atoms. This means that nitrogen, for example, becomes the nearest neighbour to the rare earth and it is mainly responsible for the crystal field. The effect of nitrogen can be estimated knowing the local configuration of interstitials around the rare earth using

$\mathrm{A}_{2}^{0}(c)=\mathrm{A}_{2}^{0}(0)+\mathrm{A}_{2}^{0}\left\{(c \zeta / 2)\left(3 \cos ^{2} \theta-1\right)\right\}$

where $c$ is the fractional occupancy of interstitial sites, $\zeta$ is the number of these sites, $\theta$ is the angle between the vector connecting the rare earth and nitrogen positions and the $c$-axis the value of $A_{2}^{0}$ is $240 \pm 60 \mathrm{Ka}_{0}^{-2}$ [15]. This accounts for the difference of sign in $\mathrm{Sm}_{2} \mathrm{Fe}_{17} \mathrm{~N}_{3}$ where the three nitrogens form a triangle around the earth perpendicular to $c$, and $\mathrm{Sm}\left(\mathrm{Fe}_{11} \mathrm{Ti}\right) \mathrm{N}$ where the two nitrogen neighbours form a dumbell along the $c$-axis.

The interstitial nitrogen can create remarkably strong uniaxial anisotropy, with a corresponding anisotropy field in excess of $20 \mathrm{~T}$. This is excessive if one is only aiming to obtain a coercivity of about 1 tesla. In fact coercivity as high as 4.4 tesla has been achieved in $\mathrm{Sm}_{3} \mathrm{Fe}_{17} \mathrm{~N}_{3}$-based magnets [16].

\section{Interstitial modification}

\subsection{Conventional gas-phase interstitial modification}

Since the discovery that nitrogen can be introduced into $\mathrm{Sm}_{2} \mathrm{Fe}_{17}$ from the gas to form $\mathrm{Sm}_{2} \mathrm{Fe}_{17} \mathrm{~N}_{3-\delta}$ with useful hard-magnetic properties [3], interstitial modification reactions have attracted much attention [17]. From the point of view of iron magnetization, the dramatic effect of the interstitial atoms is a large increase in Curie temperature (from $116^{\circ} \mathrm{C}$ for $\mathrm{Sm}_{2} \mathrm{Fe}_{17}$ to $476^{\circ} \mathrm{C}$ for $\mathrm{Sm}_{2} \mathrm{Fe}_{17} \mathrm{~N}_{3}$ ) due to the dilation of the lattice (about $6 \mathrm{vol} \%$ ). Furthermore, the anisotropy changes from easy-plane to strong easy-axis. Gas-phase interstitial modification using molecular nitrogen has been extended to intermetallics with the $\mathrm{ThMn}_{12}$ structure such as $\mathrm{Nd}\left(\mathrm{Fe}_{11} \mathrm{Ti}\right)$. Hydrogen has long been known as an interstitial in rare-earth intermetallics, but the improvement of the magnetic properties of iron-rich compounds is much less pronounced than that observed for nitrogen.

The interstitial modification of $\mathrm{Sm}_{2} \mathrm{Fe}_{17}$ using $\mathrm{N}_{2}$ and $\mathrm{H}_{2}$ is reasonably well understood. Both hydrogen and nitrogen in $\mathrm{Sm}_{2} \mathrm{Fe}_{17}$ behave as an "interaction-free lattice gas": all interatomic interaction effects can be neglected, except the site-blocking effect which excludes double occupation of interstitial sites $[18,19]$. The net reaction energy (absorption energy) $U_{0}=-57 \mathrm{~kJ} / \mathrm{mole}$ for nitrogen in $\mathrm{Sm}_{2} \mathrm{Fe}_{17}[18]$ indicates an exothermic reaction and explains the nearly complete filling of the large octahedral $9 e$ sites in $\mathrm{Sm}_{2} \mathrm{Fe}_{17}(\delta \ll 1)$. On the other hand, attempts to introduce interstitial atoms from other diatomic gases such as oxygen, fluorine, or chlorine have failed.

\subsection{Non-equilibrium interstitial modification}

Gas-phase interstitial modification using carbon from hydrocarbon gases [20] is an example of a non-equilibrium process: the gas decomposes, and carbon diffuses from a solid surface layer into the $\mathrm{Sm}_{2} \mathrm{Fe}_{17}$ lattice. Note that lattice expansions comparable to those observed for gas-solid reactions (about $5 \mathrm{vol} \%$ ) are obtained if graphite powder is used directly at low temperatures. (solid-solid interstitial modifcation [21]).

Another kind of non-equilibrium interstitial modification is the catalytic decomposition of ammonia, which involves intermediate occupation of less stable interstitial sites by nitrogen [22]. This mechanism offers a natural explanation of reported nitrogen concentrations somewhat larger than three [23], and volume expansion somewhat greater than $6 \%$.

\section{Two-phase magnets}

\subsection{Remanence enhancement}

Soft magnetic phases such as bcc iron often reduce the energy product by degrading coercivity. Nevertheless, there are two ways to avoid this destruction of coercivity. (i) The reversed soft-magnetic nucleus is prevented from propagating into the hard-magnetic phase (pinning). Model calculations [24] show that micron-size spherical soft inclusions 
in a hard matrix exhibit a very low nucleation field (they switch easily), but are not necessarily able to transmit the magnetic transversal across the phase boundary. (ii) The soft-magnetic regions are nanostructured and exchange coupled to the hard phase so the hard magntic phase stabilizes the magnetization of the whole magnet. In isotropic two-phase magnets this effect leads to remanence enhancement because it can be used to improve upon the comparatively low remance $M_{\mathrm{r}} \approx M_{0} / 2$ expected for randomly-oriented noninteracting grains. Examples are the nanocrystalline composites $\mathrm{Nd}_{2} \mathrm{Fe}_{14} \mathrm{~B} / \mathrm{Fe}_{3} \mathrm{~B}$ and $\mathrm{Sm}_{2} \mathrm{Fe}_{17} \mathrm{~N}_{3} / \mathrm{Fe}$ mentioned in the introduction. Although improved with respect to the isotropic single-phase rare earth material, the energy product of these isotropic composites does not reach the level attained in oriented rare earth magnets.

\subsection{Micromagnetic background}

Within the framework of continuum theory, the magnetic system can be described by the free energy

$F=\int\left\{A(r)\left(\frac{\nabla M}{M_{0}}\right)^{2}-K_{1}(r) \frac{(M n)^{2}}{M_{0}^{2}}-\mu_{0} M H\right\} \mathrm{d} r$

where $\boldsymbol{H}$ denotes the internal field, which is the sum of the externally applied field $\boldsymbol{H}_{\mathrm{ex}}$ and the magnetostatic "demagnetizing" field $\Delta H_{\text {int }}(M(r)) . A(r)$ is the exchange stiffness, $M(r)$ with $|\boldsymbol{M}(\boldsymbol{r})|=M_{0}$ is the local magnetization, and $K_{1}(r)$ is the first anisotropy constant.

Let us start with a perfectly aligned magnet: $M(r)=$ $M_{0}(\boldsymbol{r}) \boldsymbol{e}_{z}$. If a sufficiently-high negative external field $\boldsymbol{H}_{\mathrm{ex}}=$ $-H_{\mathrm{N}} e_{z}$ is applied, the initial magnetization state becomes unstable and magnetic reversal begins (nucleation). Nucleation is a necessary but not sufficient condition for complete magnetic reversal. It sets a lower limit to the coercive field, $H_{\mathrm{c}} \geqslant H_{\mathrm{N}}$, since there is a possibility that the reversed nucleus will not propagate due to pinning [25]. Note that $K_{2}(r)$ does not influence the nucleation field because we assume that the unit vector in the easy axis direction $\boldsymbol{n}$ is parallel to the field $H_{\mathrm{ex}}=H_{\mathrm{ex}} e_{z}$ (aligned magnet). In this case the nucleation problem reduces to a linear eigenvalue problem, but the difficulties remain considerable because of the complicated dependence of $H_{\text {int }}$ on $M(r)$. A simple, though nontrivial, approximation is to replace $\Delta H_{\text {int }}$ by a demagnetizing field $-D M_{\text {eff }} e_{z} ; D$ is the demagnetizing factor.

\subsection{Brown's paradox}

Brown's paradox is that the observed intrinsic coercivity $H_{\mathbf{c}}$ is invariably smaller than the value

$H_{\mathrm{c}} \geqslant \frac{K_{1}}{\mu_{0} M_{0}}-D M_{0}$

calculated from the theory of nucleation in homogeneous ellipsoids [26]. Two comments are necessary at this point: (i) The validity of Eq. (2) is restricted to homogeneous magnets. In fact, real systems always exhibit a certain inhomogeneity which is the origin of the much smaller coercivity observed in practice. (ii) The inequality Eq. (2) is obtained from purely energetic considerations, without referring to any particular model. This can be discussed by comparing Eq. (2) with the result of the Stoner-Wohlfarth model of coherent rotation

$H_{\mathrm{c}}=\frac{K_{1}}{\mu_{0} M_{0}}+\frac{1}{2}(1-3 D) \mu_{0} M_{0}$

Taking a macroscopically large magnet with $K_{1}=0, M_{0}=$ $2.15 \mathrm{~T}$, and $D=0.2$ (see Fig. 1) we obtain from Eq. (3) the unphysically large coercivity $\mu_{0} H_{c}=+0.43 \mathrm{~T}$. In practice, $H_{\mathrm{c}}=0$ which is consistent with the prediction $\mu_{0} H_{\mathrm{c}} \geqslant$ $-0.43 \mathrm{~T}$ from Eq. (2).

The problem, however, is to calculate the nucleation field for homogeneous and inhomogeneous magnets and to determine the incoherent modes which initiate the nucleation process. In the case of homogeneous magnets the nucleation fields are known and the corresponding nucleation modes such as curling, are well investigated [27]. As we will see, in the case of inhomogeneous magnets the answers to the problem are completely different for micronsize and nanostructed magnets, respectively.

Let us first discuss the case of microcrystalline disorder which includes, as a limit, homogeneous magnets. The main difficulty is to handle the long-range magnetostatic interaction field. The corresponding linearized eigenvalue problem consists in the diagonalization of an infinitely large, continuous matrix. To make the formalism more transparent and to emphasize the physics behind the procedure we restrict ourselves to the greatly simplified model of Fig. 2. The magnetic moment of the two hemispheres is $\boldsymbol{m}_{i}=$ $2 \pi R^{3} M_{0}\left(e_{z} \cos \theta_{i}+e_{y} \sin \theta_{i}\right) / 3$ with $i=\alpha, \beta$, and the free energy reads

$$
\begin{aligned}
\frac{F}{V}= & \left(\frac{A^{\prime}}{R^{2}}-\frac{1}{24} \mu_{0} M_{0}^{2}\right)\left(\theta_{\alpha}-\theta_{\beta}\right)^{2}+\frac{1}{2} K_{1 \alpha} \theta_{\alpha}^{2}+\frac{1}{2} K_{1 \beta} \theta_{\beta}^{2} \\
& +\frac{1}{4} \mu_{0} M_{0} H_{\text {ex }}\left(\theta_{\alpha}^{2}+\theta_{\beta}^{2}\right)
\end{aligned}
$$

$A^{\prime} \propto A$ is an effective exchange stiffness. The model has only two degrees of freedom so the nucleation problem corresponds to the diagonalization of a $2 \times 2$ matrix.

In the homogeneous case $K_{1 \alpha}=K_{1 \beta}=K_{1}$ the expression Eq. (4) is diagonalized by the two eigenvectors (nucleation modes) $\theta=\theta_{\alpha}+\theta_{\beta}$ [Fig. 2(b)] and $\theta^{\prime}=\theta_{\alpha}-\theta_{\beta}$ [Fig. 2(c)]. $\theta$ describes coherent rotation and the nucleation field (eigenvalue), at which the quadratic form Eq. (4) ceases to be

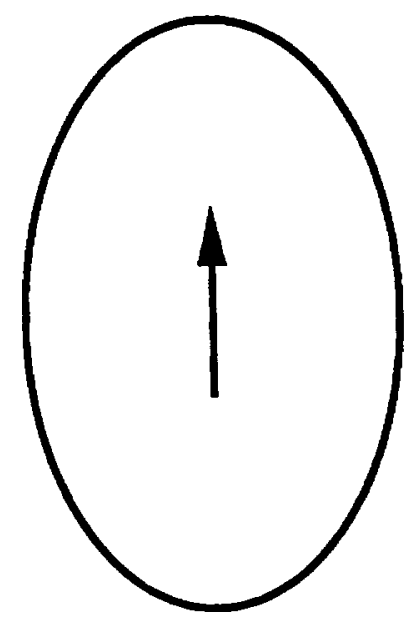

Fig. 1. Ellipsoid with $c / a=1.75$ and $D=0.2$. If the magnetization is assumed to remain parallel (coherent rotation), the stray field energy yields a finite coercivity which is not observed for macroscopic magnets. 


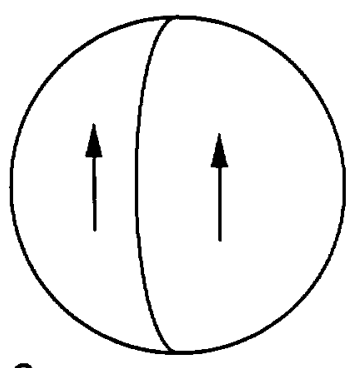

a

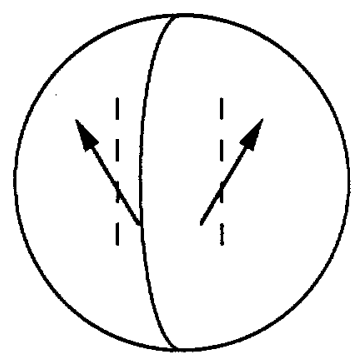

C

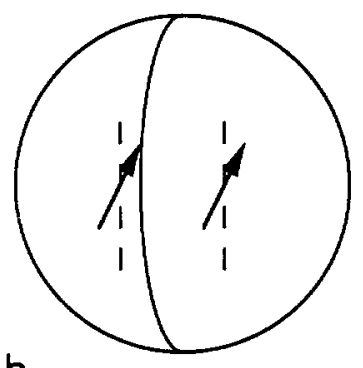

b

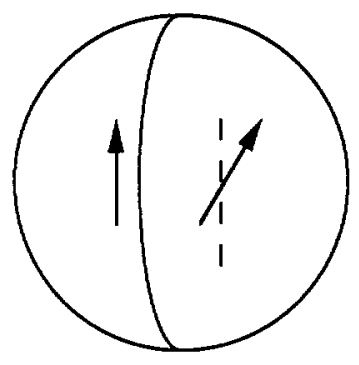

d
Fig. 2. A simplified nucleation model. (a) Initial stage. (b) Coherent rotation. (c) Incoherent rotation. (d) Localized nucleation mode.

positive definite, is given by Eq. (3) with $D=1 / 3$. The mode $\theta^{\prime}$ describes an incoherent, curling-like nucleation process with

$H_{\mathrm{N}}=\frac{K_{1}}{\mu_{0} M_{0}}+\frac{8 A^{\prime}}{\mu_{0} M_{0} R^{2}}-\frac{1}{3} M_{0}$

For very small, single-domain particles the field given by Eq. (5) is very high so magnetic reversal is actually realized by coherent rotation. On the other hand, in the limit $R \rightarrow \infty$ the exchange term is negligible and Brown's inequality Eq. (2) becomes an equality. Note that this behaviour has been confirmed by exact calculations [27].

In the inhomogeneous case $K_{1 \beta} \ll K_{1 \alpha}$ the nucleation mode is localized [Fig. 2(d)] and the nucleation fields reads

$H_{\mathrm{N}}=\frac{K_{1 \beta}}{\mu_{0} M_{0}}+\frac{4 A^{\prime}}{\mu_{0} M_{0} R^{2}}-\frac{1}{6} M_{0}$

Equation (6) resolves Brown's paradox for the model of Fig. 2. The soft hemisphere switches at a very low field unless its magnetization direction is stabilized by exchange coupling in a particle of very small radius.

Anisotropy fields of modern rare earth magnets exceed typical magnetostatic fields by an order of magnitude so magnetostatic fields cannot be held responsible for the low coercivity observed in these materials. In this limit the magnetostatic interaction in Eq. (1) can be neglected and it becomes clear directly that violations of Eq. (2) must be due to the spatial variation of anisotropy and exchange.

\subsection{Aligned two-phase magnets}

To calculate the nucleation field for reversal of aligned nanostructured magnets, we start with the free energy Eq. (1) and use the identity

$M(\boldsymbol{r})=M_{0}(\boldsymbol{r})\left(m_{x}(\boldsymbol{r}) \boldsymbol{e}_{x}+m_{y}(\boldsymbol{r}) \boldsymbol{e}_{y}+\sqrt{1-m_{x}^{2}-m_{y}^{2}} \boldsymbol{e}_{z}\right)$

to expand the free energy density with respect to the small transverse components $m_{x}, m_{y} \ll 1$. This yields the micromagnetic eigenvalue equation

$\nabla(A(\boldsymbol{r}) \nabla \boldsymbol{m})-K_{1}(\boldsymbol{r}) \boldsymbol{m}=-\frac{1}{2} \mu_{0} M_{0}(\boldsymbol{r}) H_{\mathrm{N}} \boldsymbol{m}$ with $\boldsymbol{m}=m_{x}(\boldsymbol{r}) \boldsymbol{e}_{x}+m_{y}(\boldsymbol{r}) \boldsymbol{e}_{y}$. Apart from its vector character and a minor generalization of the boundary condition, Eq. (8) corresponds to Schrödinger's equation for a particle moving in a three-dimensional potential $2 K_{1}(\boldsymbol{r}) / \mu_{0} M_{0}(\boldsymbol{r})$ [16]. This allows us to apply ideas familiar from quantum mechanics to discuss micromagnetics $[24,28]$. In particular, the nucleation field corresponds to the quantum-mechanical ground-state energy, and the small transverse magnetization or nucleation mode $\boldsymbol{m}$ is the analog of the wave function $\Psi$.

The hard and soft phases can have different values of the parameters $A, M_{0}$, and $K_{1}$ denoted by the suffixes $\mathrm{h}$ and $\mathrm{s}$. First we consider an ideally soft inclusion (diameter $D$, $K_{\mathrm{s}}=0$ ) in a hard matrix. It turns out [28] that the nucleation field reaches a plateau below $D \approx \delta_{\mathrm{h}}$, where $\delta_{\mathrm{h}}$ is the Bloch wall width of the hard phase (Fig. 3). This plateau corresponds to complete exchange coupling. Taking values appropriate to $\mathrm{Sm}_{2} \mathrm{Fe}_{17} \mathrm{~N}_{3}$, the intermetallic with the most favourable combination of magnetization and anisotropy (Table 1), we see that inclusions with a diameter $D \approx 7 \mathrm{~nm}$ retain a coercivity as high as $\mu_{0} H_{\mathrm{N}}=7 \mathrm{~T}$. For larger inclusions the coercivity falls off as $1 / D^{2}$; inhomogeneous magnetostatic fields can initiate nucleation at diameters greater than $20 \mathrm{~nm}$.

For sufficiently small reverse fields $|H|<H_{\mathrm{N}}(D)$ the single soft inclusion is perfectly aligned and slightly enhances the remanence provided $M_{\mathrm{s}}>M_{\mathrm{h}}$. To obtain a significant remanence enhancement it is necessary to generalize to large numbers of spherical inclusions per unit volume, but when the distance between neighbouring inclusions becomes too small, the soft regions interact and coercivity is destroyed. In the case of well-separated inclusions, the lowest-lying magnetization mode which is responsible for nucleation decreases exponentially in the hard region. But when the distance between the soft inclusions is small, the magnetization modes can "tunnel" through the hard region which no longer acts as an effective potential barrier. In fact, this micromagnetic "exchange interaction" can reduce the nucleation field considerably when the thickness of the hard region is less than $\delta_{\mathrm{h}}$.

In the plateau region (Fig. 3), where the soft regions are very small, the problem can be treated in perturbation theory [28]. As in quantum mechanics, the lowest order

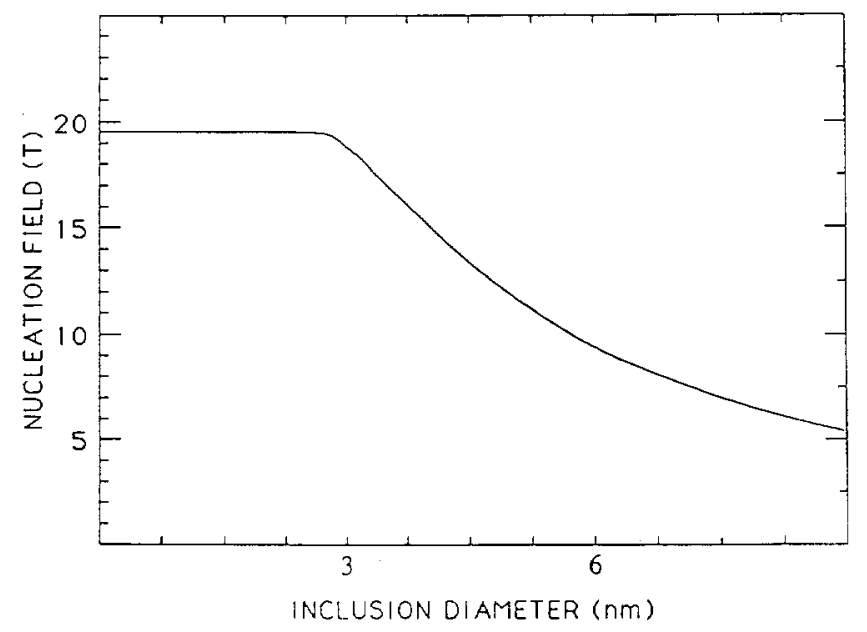

Fig. 3. Nucleation field $H_{\mathrm{N}}$ as function of $D$, the diameter of a soft inclusion in a hard matrix. The values assumed are for the $\mathrm{Sm}_{2} \mathrm{De}_{17} \mathrm{~N}_{3} / \mathrm{Fe}$ system: $\mu_{0} M_{\mathrm{s}}=2.15 \mathrm{~T}, \mu_{0} M_{\mathrm{h}}=1.55 \mathrm{~T}, A_{\mathrm{s}} / A_{\mathrm{h}}=1.5, K_{\mathrm{s}}=0$ and $K_{\mathrm{h}}=$ $12 \mathrm{~kJ} / \mathrm{m}^{3}$. 
eigenvalue correction is obtained by using the normalized unperturbed function $\Psi_{0}$. This yields the nucleation field $\mu_{0} H_{\mathrm{N}}=2\left(f_{\mathrm{s}} K_{\mathrm{s}}+f_{\mathrm{h}} K_{\mathrm{h}}\right) /\left(f_{\mathrm{s}} M_{\mathrm{s}}+f_{\mathrm{h}} K_{\mathrm{h}}\right)$ where $f_{\mathrm{s}}$ and $f_{\mathrm{h}}=(1$ $-f_{\mathrm{s}}$ ) are the volume fractions of the soft and hard phases, respectively. If we neglect further pinning of the nucleus, we obtain a rectangular hysteresis loop with $H_{\mathrm{c}}=H_{\mathrm{N}}$ and $M_{\mathrm{r}}=\left\langle M_{0}(r)\right\rangle$. The energy product depends on the shape of the magnet, but the optimum value is given by $\mu_{0} M_{\mathrm{r}}^{2} / 4$ for $H_{\mathrm{N}}>M_{\mathrm{r}} / 2$ and $\mu_{0} H_{\mathrm{N}} M_{\mathrm{r}} / 2$ for $H_{\mathrm{N}}<M_{\mathrm{r}} / 2$. Putting $K_{\mathrm{s}}=0$ we find the highest energy product obtained for $H_{\mathrm{N}}=\mathrm{M}_{\mathrm{r}} / 2$

$(B H)_{\max }=4 \mu_{0} M_{\mathrm{s}}^{2}\left(1-\frac{\mu_{0}\left(M_{\mathrm{s}}-M_{\mathrm{h}}\right) M_{\mathrm{s}}}{2 K_{\mathrm{h}}}\right)$

Due to the large $K_{\mathrm{h}}$, the second term in the bracket is small so the energy product approaches the ultimate value of $\mu_{0} M_{\mathrm{s}}^{2}$. The corresponding volume fraction of the hard phase is $f_{\mathrm{h}}=\mu_{0} M_{\mathrm{s}}^{2} / 4 K_{\mathrm{h}}$.

If we consider the $\mathrm{Sm}_{2} \mathrm{Fe}_{17} \mathrm{~N}_{3} / \mathrm{Fe}_{65} \mathrm{Co}_{35}$ system and take values $\mu_{0} M_{\mathrm{s}}=2.43 \mathrm{~T}, \mu_{0} M_{\mathrm{h}}=1.55 \mathrm{~T}$, and $K_{\mathrm{h}}=12 \mathrm{MJ} / \mathrm{m}^{3}$, we obtain a theoretical energy product of $1090 \mathrm{~kJ} / \mathrm{m}^{3}$ (137 MGOe) for a volume fraction of only $9 \%$ of the hard phase. It is remarkable that these optimum magnets are almost entirely composed of $3 d$ metals, with only about $2 \mathrm{wt} \%$ samarium.

The nucleation field is independent of the shape of the soft regions, so long as their size lies in the plateau region (Fig. 3). The hard regions act as a skeleton to stiffen the magnetization direction of the soft regions. The practical problem however is to realize a structure where the soft regions are sufficiently small to avoid nucleation at small fields while having the hard regions crystallographically oriented. One conceivable solution is a disordered twophase magnet Fig. 4 with a common $c$ axis throughout the hard regions, but it is difficult to see how this might be achieved. A more realistic possibility may be a multilayered structure of alternating soft and hard magnetic layers (Fig. 5). Assuming a uniform demagnetizing field, a "megajoule magnet" using $\mathrm{Sm}_{2} \mathrm{Fe}_{17} \mathrm{~N}_{3}$ and $\mathrm{Fe}_{65} \mathrm{Co}_{35}$ is obtained for layer thicknesses $\lambda_{\mathrm{h}}=2.4 \mathrm{~nm}$ and $\lambda_{\mathrm{c}}=\lambda_{\mathrm{s}}=9.0 \mathrm{~nm}$ [16]. The form of the magnet must of course correspond to the

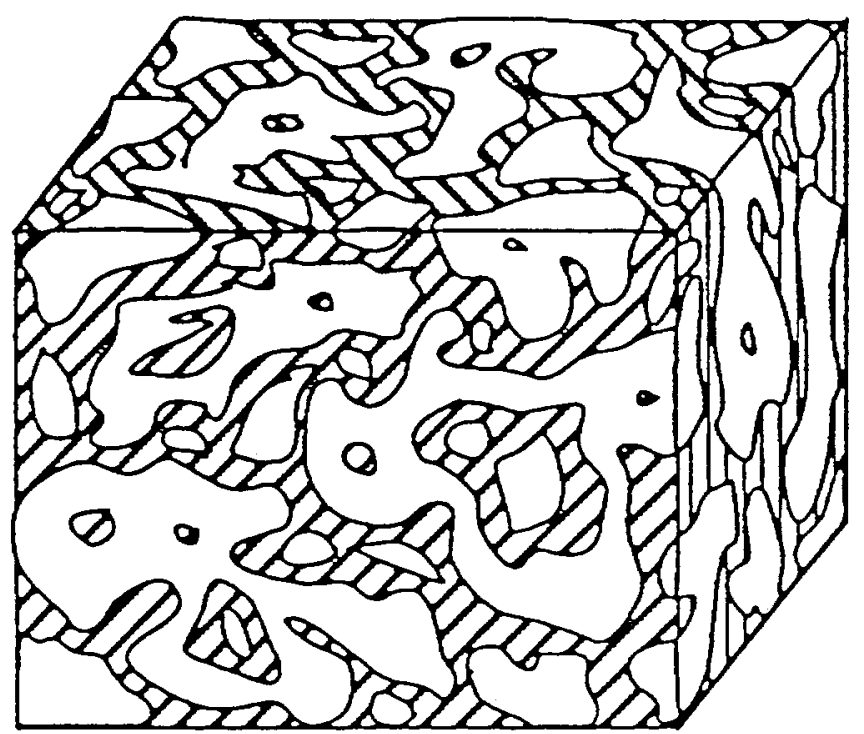

Fig. 4. An oriented disordered two-phase magnet with common $c$ axis. The size of the soft and hard regions is smaller than the Bloch wall thickness $\mathrm{dh}$ of the hard phase

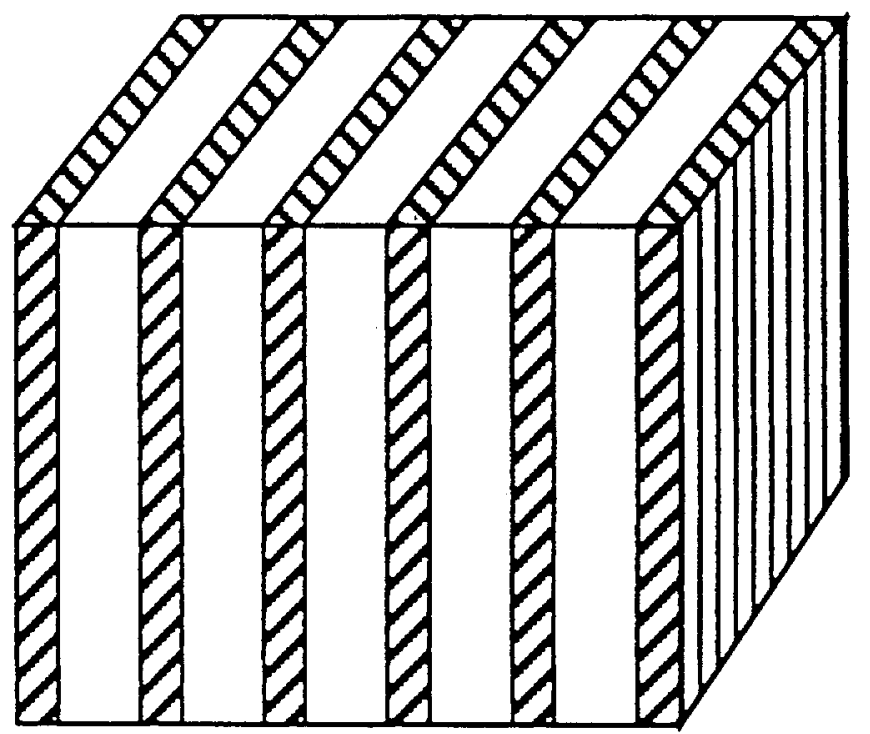

Fig. 5. A multilayer structure composed of alternating hard and soft magnetic regions. The multilayer periodicity should not exceed about ten nanometers.

optimum operating point on the $B H$-curve; it should approximate an ellipsoid with $c / a=0.55$.

Equation (1) is based on classical micromagnetic considerations; the sizes of the hard and soft regions must be large compared to atomic dimensions so that a continuum model can be applied. However, due to the small prefactor $\left(M_{\mathrm{s}}-M_{\mathrm{h}}\right) / M_{\mathrm{s}}, 0.28$ for the $\mathrm{Sm}_{2} \mathrm{Fe}_{17} \mathrm{~N}_{3} / \mathrm{Fe}$ system, the energy product is not much affected if the fraction of the hard phase is increased. We have still an energy product of almost $800 \mathrm{~kJ} / \mathrm{m}^{3}$ (100 MGOe) in the $\mathrm{Sm}_{2} \mathrm{Fe}_{17} \mathrm{~N}_{3} / \mathrm{Fe}$ system when $f_{\mathrm{h}} \approx 30 \%$, and we can use extra hard material to outweigh quantum-mechanical size effects.

\section{Conclusions}

Scope for significant improvements in the energy product of permanent magnets based on the appearance of a new rare earth iron intermetallic phase is very limited, although new materials could offer better temperature stability, yet higher anisotropy field, improved corrosion resistance or more favourable performance/cost ratio. A radically new approach is called for if substantial improvement in the energy product is ever again to be achieved. Here we have outlined one such approach, involving exchange hardening in nanoscale combinations of a soft phase and an oriented hard phase. If it is possible to structure these materials according to the principles we have outlined, the ultimate room temperature permanent magnets with energy products approaching $1 \mathrm{MJ} / \mathrm{m}^{3}$ could be achieved.

\section{Acknowledgements}

This work was supported by the BRITE/EURAM Programme of the European Commission.

\section{References}

1. Sagawa, M., Fujimura, S., Togawa, N., Yamamoto, H. and Matsuira, Y., J. Appl. Phys. 55, 2083 (1984)

2. Croat, J. J., Herbst, J. F., Lee, R. W. and Pinkerton, F. E., J. Appl. Phys. 55, 2078 (1984). 
3. Coey, J. M. D. and Sun, H., J. Magn. Magn. Mater. 87, L251 (1990).

4. Krönmuller, H., in: "Supermagnets, Hard Magnetic Materials" (Edited by G. J. Long and F. Grandjean) (Kluwer, Dordrecht 1991), p. 461.

5. Sagawa, M., Hirosawa, S., Yamamoto, H., Fujimura, S. and Matsuura, Y., Jpn. J. Appl. Phys. 26, 785 (1987).

6. Coehoorn, R., de Mooij, D. B., Duchateau, J. P. W. B. and Buschow, K. H. J., J. Physique. 49, C8-669 (1988).

7. Ding, J., McCormick, P. G. and Street, R., J. Magn. Magn. Mater., in press (1993).

8. Kneller, E. and Hawig, R., Festschrift volume for Prof. H. Warlimont, Bochum 1991 p. 6.

9. Hernando, A., J. Magn. Magn. Mater., in press (1992).

10. Moruzzi, V. L., Marcus, P. M., Schwarz, K. and Mohn, P., Phys. Rev. B34, 1784 (1986).

11. Akai, M., Akai, H. and Kanamori, J., J. Phys. Soc. Japan 56, 1064 (1987).

12. Komuro, M., Kozono, Y., Hanazono, M. and Sugita, Y., J. Appl. Phys., 67, 5126 (1990); Takahashi, H., Mitsuoka, K., Komuro, M. and Sugita, Y., J. Appl. Phys. 73, 6060 (1993).

13. Akai, M., Akai, H. and Kanamori, J., Phys. Soc. Japan 56, 1064 (1987).

14. Coehoorn, R., Buschow, K. H. J., Dirken, M. W. and Thiel, R. C., Phys. Rev. B42, 4645 (1990).
15. Skomski, R., Kuz'min, M. D. and Coey, J. M. D., J. Appl. Phys. 73, 6934 (1993).

16. Kuhrt, C., O'Donnell, K., Katter, M., Wecker, J., Schnitzke, K. and Schultz, L., Appl. Phys. Lett. 60, 3316 (1992).

17. Coey, J. M. D., Physica Scripta T39, 21 (1991).

18. Coey, J. M. D., Skomski, R. and Wirth, S., IEEE Trans. Mag. 28, 2332 (1992).

19. Skomski, R. and Coey, J. M. D., J. Appl. Phys., 73, 7602 (1993).

20. Coey, J. M. D., Sun, H., Otani, Y. and Hurley, D. P. F., J. Magn. Magn. Mater. 98, 76 (1991).

21. Skomski, R., Murray, C., Brennan, S. and Coey, J. M. D., J. Appl. Phys., 73, 6940 (1993).

22. Skomski, R., Brennan, S. and Coey, J. M. D., Phys. Stat. Sol. (a) 1993 (in press).

23. Iriyama, T., Kobayashi, K., Fukuda, T., Kato, H. and Nakagawa, Y., IEEE Trans. Mag. 28, 2326 (1992).

24. Skomski, R., Phys. Stat. Sol. (b) 174, K 77 (1992).

25. Givord, D., Lu, Q. and Rossignol, M. F., in: "Science and Technology of Nanostructured Magnetic Materials" (Edited by G. C. Hadjipanayis and G. A. Prinz) (Plenum, New York, 1991), p. 635.

26. Brown, W. F., Rev. Mod. Phys. 17, 15 (1945).

27. Aharoni, A., Rev. Mod. Phys. 34, 227 (1962).

28. Skomski, R. and Coey, J. M. D., IEEE Trans. Magn., 1993 (in press). 